\title{
Efficacy of Aquatic Treadmill Training on Gait Symmetry and Balance in Subacute Stroke Patients
}

\author{
Mi Eun Lee, MD, Geun Yeol Jo, MD, PhD, Hwan Kwon Do, MD, Hee Eun Choi, MD, Woo Jin Kim, MD \\ Department of Physical Medicine and Rehabilitation, Inje University Haeundae Paik Hospital, \\ Inje University College of Medicine, Busan, Korea
}

\begin{abstract}
Objective To determine the efficacy of aquatic treadmill training (ATT) as a new modality for stroke rehabilitation, by assessing changes in gait symmetry, balance function, and subjective balance confidence for the paretic and non-paretic leg in stroke patients.

Methods Twenty-one subacute stroke patients participated in 15 intervention sessions of aquatic treadmill training. The Comfortable 10-Meter Walk Test (CWT), spatiotemporal gait parameters, Berg Balance Scale (BBS), and Activities-specific Balance Confidence scale (ABC) were assessed pre- and post-interventions.

Results From pre- to post-intervention, statistically significant improvements were observed in the CWT $(0.471 \pm$ 0.21 to $0.558 \pm 0.23, \mathrm{p}<0.001)$, BBS $(39.66 \pm 8.63$ to $43.80 \pm 5.21, \mathrm{p}<0.001)$, and $\mathrm{ABC}(38.39 \pm 13.46$ to $46.93 \pm 12.32$, $\mathrm{p}<0.001)$. The step-length symmetry $(1.017 \pm 0.25$ to $0.990 \pm 0.19, \mathrm{p}=0.720)$ and overall temporal symmetry $(1.404 \pm$ 0.36 to $1.314 \pm 0.34, \mathrm{p}=0.218$ ) showed improvement without statistical significance.

Conclusion ATT improves the functional aspects of gait, including CWT, BBS and ABC, and spatiotemporal gait symmetry, though without statistical significance. Further studies are required to examine and compare the potential benefits of ATT as a new modality for stroke therapy, with other modalities.
\end{abstract}

Keywords Stroke, Rehabilitation, Gait, Hydrotherapy

\section{INTRODUCTION}

According to the 57th annual statistical report of the Korean Ministry of Health and Welfare (of 2011), stroke was the second most common cause of death due to chronic illness, with a prevalence rate of $3.9 \%$. In the United States, stroke is a common cause of long-term disability [1]. Among the serious neurological deficits caused by stroke, most significant is the loss of motor functions, inducing paralysis, pathologic reflexes, and spasticity,

Received May 25, 2016; Accepted September 9, 2016

Corresponding author: Woo Jin Kim

Department of Physical Medicine and Rehabilitation, Inje University Haeundae Paik Hospital, Inje University College of Medicine, 875 Haeun-daero, Haeundae-gu, Busan 48108, Korea. Tel: +82-51-797-3250, Fax: +82-51-797-0298, E-mail: cyctaz@hanmail.com

ORCID: Mi Eun Lee (http://orcid.org/0000-0002-9807-0108); Geun Yeol Jo (http://orcid.org/0000-0002-5726-060X); Hwan Kwon Do (http://orcid. org/0000-0002-5862-4233); Hee Eun Choi (http://orcid.org/0000-0002-8753-929X); Woo Jin Kim (http://orcid.org/0000-0001-6172-2750).

@ This is an open-access article distributed under the terms of the Creative Commons Attribution Non-Commercial License (http://creativecommons.org/ licenses/by-nc/4.0) which permits unrestricted noncommercial use, distribution, and reproduction in any medium, provided the original work is properly cited. Copyright (c) 2017 by Korean Academy of Rehabilitation Medicine 
which interfere with independent mobility [2]. The representative features of hemiplegic gait include low gait velocity and asymmetry of step length, stance phase time, swing phase time, and limb joint angles [3]. These lead to balance impairment with increased risk of falling, and limitations on activities of daily living and participation in community social activities.

Conventional rehabilitation after stroke improves the general locomotor functions. One of the traditional modalities, treadmill training has been beneficial in its effectiveness for walking ability and balance. Body weight supported treadmill training (BWSTT) is one of the preferred therapies for patients who cannot walk independently, and its therapeutic effects have been demonstrated in several studies, through improvements in balance, balance confidence, gait speed, and body coordination $[4,5]$.

Underwater or aquatic exercise has been considered a major therapeutic modality for many decades. The essential physical properties of water are density, hydrostatic pressure, buoyancy, viscosity, and thermodynamics [6]. Buoyancy has great therapeutic utility by offsetting gravity, so that only the muscle torque forces act on the limbs. Viscosity and hydrostatic pressure are able to support a body that lacks balance; moreover, these properties reduce the fear of falling, and encourage balance confidence. Depending on the water level of the patient's immersed body, the effect of weight support varies. Xiphoid-depth immersion is equivalent to the effect of $60 \%$ or more offloading, depending on arm position [6]. Chu et al. [7] reported improvements in gait velocity after 8 weeks of aquatic exercise in stroke patients.

Aquatic treadmill training (ATT) employs underwater treadmill that combines the benefits of water immersion with the advantages of continuous and rhythmic treadmill gait training. The body weight supporting effect due to buoyancy is the key beneficial feature of the water in ATT, similar to its effect in BWSTT.

Studies on the effects of ATT have been reported for the healthy elderly, and for patients with spinal cord injury, osteoarthritis, Parkinson's disease, and stroke. A study on 10 stroke patients by Yoo et al. [8] reported that changes in blood pressure and heart rate during underwater treadmill training were lesser than during land treadmill training, thus reducing the workload of the cardiovascular system. Jung et al. [9] reported that the application of additional weight on the affected leg reduced unwanted flotation in chronic stroke patients during ATT, leading to increased stance stability.

Efficacy of ATT on changes in the spatiotemporal measurement, balance, and balance confidence in subacute phase stroke patients, has never been reported in literature. This study aims to evaluate the changes in gait symmetry, balance function, and subjective balance confidence for the paretic and non-paretic legs in stroke patients, before and after aquatic treadmill intervention, and to further determine the efficacy of ATT as a new modality for stroke rehabilitation.

\section{MATERIALS AND METHODS}

\section{Study design}

This study compared the pre- and post-intervention spatiotemporal data of gait, balance, and balance confidence, in a single group of patients diagnosed with stroke. The study was approved by the Institutional Review Boards of Inje University College of Medicine and Inje University Haeundae Paik Hospital, and all the patients gave consent to participate in this study.

\section{Participants}

Totally, 23 subacute hemiparetic stroke patients, with onset duration ranging from 3 weeks to 6 months, were recruited. The patients were all admitted to the Department of Physical Medicine and Rehabilitation of Inje University Haeundae Paik Hospital for comprehensive rehabilitation. The study enrollment criteria included (1) independent walk more than $10 \mathrm{~m}$ with or without the use of orthosis, (2) Mini-Mental State Examination (MMSE) score $>24$, and (3) no or mild spasticity, with Modified Ashworth Scale score of $\leq 2$ in the paretic limb. The exclusion criteria were (1) history of previous stroke, (2) skin disease such as pressure sores, (3) severe neurologic or musculoskeletal disease preventing participation, and (4) aphasia.

\section{Intervention}

The participants performed 15 intervention sessions of ATT over 3 weeks. This experiment employed the aquatic treadmill training device, Aquatrac-2000 (NARAMED, Gwangju, Korea) (Fig. 1). The sessions were held 5 days per week, each of 20 minutes duration. Each session 


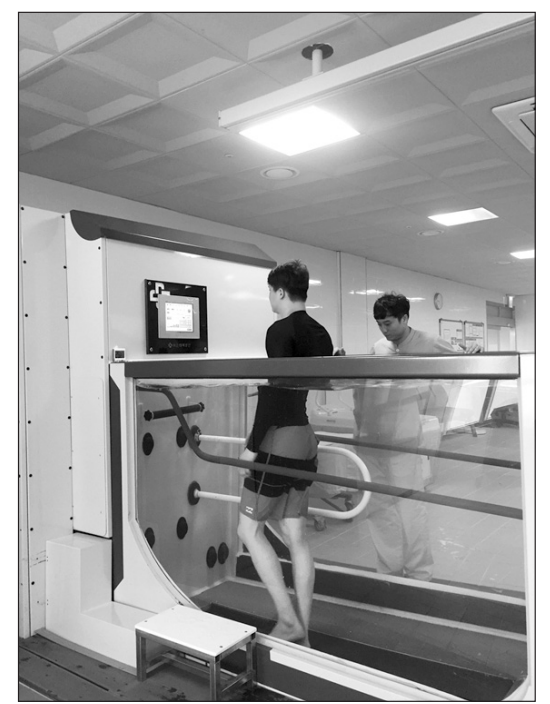

Fig. 1. Aquatic treadmill intervention under the supervision of a physical therapist.

comprised 4 phases at different velocities, at 5-minute duration per phase. The first phase started at a self-selected treadmill speed. The treadmill speed was increased by $0.5 \mathrm{~km} / \mathrm{hr}$ increments in each of the $2 \mathrm{nd}$ and $3 \mathrm{rd}$ phases, and was reduced to speed of the 1st phase during the 4 th phase. Since none of the participants had experience with ATT, 10 minutes was assigned prior to the actual intervention, for adapting to the machine and help in selecting the most comfortable treadmill speed. The level of the water in the treadmill was matched to each participant's xiphoid process, providing support for $60 \%$ or more of the body weight [6]. During treadmill training, two breaks of less than 2 minutes were allowed. For the safety of the participants, heart rate and blood pressure were measured before and after each intervention. Participants performed the intervention without holding the hand rails of the machine. An occasional verbal cue was provided by the physical therapist at standby. Simultaneously, conventional rehabilitation treatments were provided to all the participants. The participants had similar functional ability, and the treatment was composed of two physical therapy sessions of 30 minutes and two occupational therapy sessions of 30 minutes per day, 5 days a week. The physical therapy session included stretching, muscle-strengthening, and gait training, while the occupational therapy session consisted of fine motor activities for the affected upper limbs and activities of daily living training.

\section{Data collection}

Pre- and post-intervention data were collected. For the primary outcomes, Comfortable 10-Meter Walk Test (CWT) and the spatiotemporal ratio of gait were measured using gait analysis. Step length, stance time, and swing time data were assessed and measured for paretic and non-paretic legs using a motion analysis system, and gait symmetry was calculated from the ratios for each of the data. For the secondary outcomes, the Berg Balance Scale (BBS) and the Activities-specific Balance Confidence scale $(A B C)$ were measured. BBS is the most widely used scale for objective balance function, comprising of 14 items using simple tools such as a chair and ruler [10]. The $\mathrm{ABC}$ is a subjective questionnaire with 16 questions regarding balance confidence, whose high reliability was confirmed by Botner et al. [11].

\section{CWT}

The CWT is a valid and reliable measure of self-selected overground gait velocity for short distances in stroke patients [12]. Velocity of the middle $10 \mathrm{~m}$ is measured from 14 $\mathrm{m}$, walked at a comfortable self-selected speed, and with at least 30 seconds rest between trials. The mean value from the 3 trials was calculated for the data analysis.

\section{Spatiotemporal gait parameters}

Spatiotemporal gait parameters were measured using the Vicon motion analysis system (Vicon Motion Systems, Oxford, UK). Sixteen retro-reflective, round 2.5$\mathrm{cm}$ markers were placed on the left and right lower limbs and the spine. The markers are placed bilaterally on the anterior superior iliac spine (ASIS), posterior superior iliac spine (PSIS), lower $1 / 3$ rd of lateral mid-femur, lateral knee joint line, lower $1 / 3$ rd of lateral tibia, lateral malleolus, calcaneus, and dorsal second metatarsal-phalangeal joint (Fig. 2A). The subjects walked at a self-selected and most comfortable velocity on a $12-\mathrm{m}$ footpath, and data were collected for 5 repeated trials [13] (Fig. 2B). Step length, stance time, and swing time measurements were recorded from the measured data. To compare the gait symmetry of the left and right lower limbs, symmetry ratio values for each set of data were calculated using the following equation:

Symmetry ratio $=\frac{\text { paretic side }(\text { parameter value })}{\text { non-paretic side (parameter value })}$ 


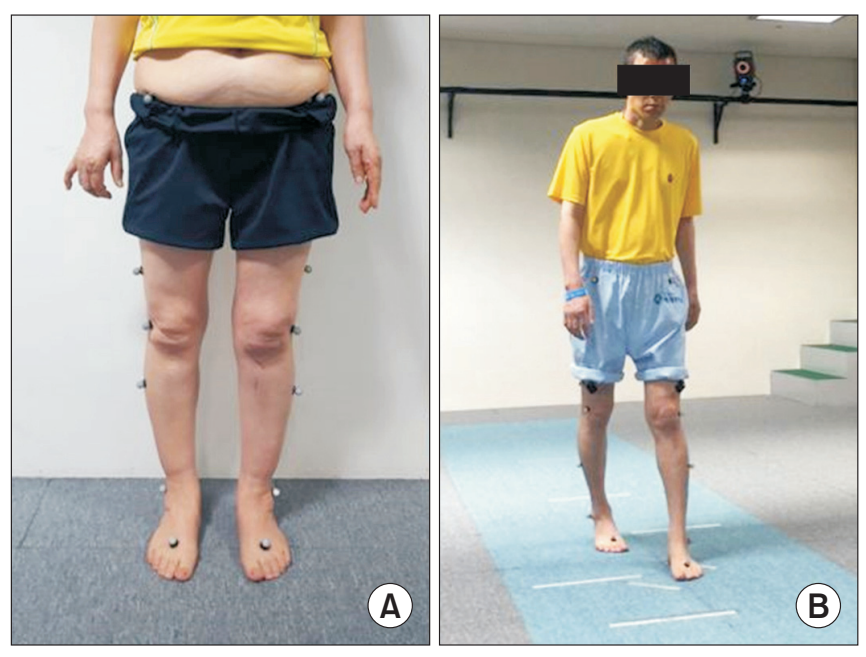

Fig. 2. Measuring Comfortable Walk Test and spatiotemporal gait parameters using motion analysis system. (A) Location of the markers for gait analysis. (B) The subject walking at a self-selected and most comfortable velocity on a 12-meter pathway.

Gait symmetry increased with values closer to 1 , and decreased with values further from 1 . A greater absolute value of the symmetry ratio implies larger parameter of the paretic side. The ratios of step length, stance time, and swing time are defined as follows:

Step length symmetry $=\frac{\text { paretic step length }}{\text { non-paretic step length }}$

Swing symmetry $=\frac{\text { paretic swing time }}{\text { non-paretic swing time }}$

Stance symmetry $=\frac{\text { paretic stance time }}{\text { non-paretic stance time }}$

Swing-stance symmetry $=\frac{\text { swing time }}{\text { stance time }}$

Overall temporal symmetry= $\frac{\text { paretic swing-stance symmetry }}{\text { non-paretic swing-stance symmetry }}$

The magnitude of symmetry was calculated by setting the numerator in the above formulae to the higher value from among the values obtained for the right and left lower limbs, irrespective of paretic side [14]. In addition, when the observed value of the symmetry ratio belonged to asymmetry, the 'direction of asymmetry' was deter- mined based on which value was greater between the paretic and non-paretic values; when the paretic value was greater, it was named 'paretic' (P); when the non-paretic value was greater, it was named 'non-paretic' (NP).

\section{$B B S$}

The BBS is an objective measurement for dynamic balance, through performance of 14 functional tasks. Items on the BBS were scored on a scale of 0 to 4 points, with a maximum summed score of 56 . The BBS has high intrarater and test-retest reliability. Minimum detectable change for the BBS for persons with stroke is defined as a 6-point difference [15]. In a study of 655 patients by Blum and Korner-Bitensky [16] on the usefulness of the BBS in stroke rehabilitation, excellent internal consistency (IC, $0.92-0.98)$, inter-rater reliability $(0.95-0.98) /$ intra-rater reliability (0.97), and test-retest reliability $(0.98)$ were obtained.

\section{$A B C$}

The ABC Scale is a self-reported questionnaire, in which participants subjectively rate their balance confidence in performing 16 activities on a scale from $0 \%$ (no confidence) to $100 \%$ (complete confidence). The average scores of the 16 items were used. For inability to perform activities due to hospitalization after the stroke attack and never having been outside circumstances, patients were asked to imagine performing the task and rate their confidence scale. The ABC has high test-retest reliability, excellent IC, and moderate correlation with the BBS, CWT, and Fast Walk Test [4]. In stroke patients, Botner et al. [11] confirmed the internal consistency of the ABC scale of 0.94 , and intraclass correlation coefficient (ICC) for the test-retest reliability of 0.85 ( $95 \%$ confidence interval, 0.68-0.93).

\section{Data and statistical analysis}

Demographic data, including age, stroke duration, and MMSE score, were collected and recorded. Changes in CWT, step length, stance time, swing time, BBS, and ABC scores were assessed for pre- to post-intervention differences using a paired t-test. The p-value of 0.05 was set as the cut-off value. All statistical analyses were performed using the SPSS ver. 20.0 (IBM, Armonk, NY, USA).

Among the pre-intervention measures, the cut-point of swing phase SR was set at 1.06, while the cut-point of step 
length SR was set at 1.08 [14]. Patients with pre-intervention symmetry ratios greater than these values were classified as asymmetric. For post-intervention measures, the significant minimal detectable change (MDC) values were set at swing SR of 0.26 , and step SR of 0.19 [17]. Patients with change scores greater than the MDC were categorized as either 'Improved' or 'Worse', depending on whether the post-intervention SR was closer to or further away from 1.0, respectively. As all others were categorized as 'No change', they were further divided into 'No change-symmetric' and 'No change-asymmetric', based on their pre-intervention SR. The above process categorized the patients into 4 groups, based on the changes in swing and step SR: 'Improved, 'No change-asymmetric', 'No change-symmetric', and 'Worse.' The number of patients in the groups 'Improved' and 'Worse' were classified by the change in direction of the swing and step asymmetry, from pre- to post-intervention. If the direc-

Table 1. Participant characteristics $(n=21)$

\begin{tabular}{lc}
\hline \multicolumn{1}{c}{ Characteristic } & Value \\
\hline Age $(\mathrm{yr})$ & $56.95 \pm 12.8$ \\
\hline Gender (male:female) & $13: 8$ \\
Stroke onset duration (day) & $63.33 \pm 54.4$ \\
MMSE & $28.19 \pm 2.23$ \\
\hline
\end{tabular}

Values are presented as mean \pm standard deviation. MMSE, Mini-Mental State Examination. tion of asymmetry at pre- to post-intervention was the same, the patients were classified as 'No change'. The number of patients with a change in symmetry direction were categorized as either 'PtoNP' (if the larger paretic value at pre-intervention changed to a larger non-paretic value at post-intervention), or 'NPtoP' (if the larger nonparetic value at pre-intervention changes to larger paretic value at post-intervention) [18].

\section{RESULTS}

Of the 21 subjects enrolled in this study, there were 2 withdrawals: one was discharged early and lost to followup, while the other discontinued ATT due to an inflamed ingrown nail. Patient characteristics are shown in Table 1. The mean age was $56.95 \pm 12.8$ years. There were 13 female and 8 male patients. The mean duration since stroke onset was $63.33 \pm 54.4$ days, and the mean MMSE score was $28.19 \pm 2.23$.

Pre- and post-intervention data for paretic and nonparetic legs were compared for the spatiotemporal measurement of gait analysis (Table 2). For both non-paretic and paretic legs, there was increase in the step length, whereas stance and swing time decreased. Changes in the pre- and post-intervention step length and stance time of the non-paretic leg were found to be significant ( $p=0.024$ and $\mathrm{p}=0.006$, respectively).

Table 2. Difference in spatiotemporal factors for each leg

\begin{tabular}{ccccccccc}
\hline \multirow{2}{*}{ Variable } & \multicolumn{3}{c}{ Paretic leg } & \multicolumn{3}{c}{ Non-paretic leg } \\
\cline { 2 - 4 } \cline { 6 - 8 } & Pre-intervention & Post-intervention & p-value & & Pre-intervention & Post-intervention & p-value \\
\hline Step length (m) & 0.351 & 0.406 & 0.060 & & 0.353 & $0.414^{*}$ & 0.024 \\
Stance time (s) & 1.118 & 1.018 & 0.094 & & 1.214 & $1.092^{*}$ & 0.006 \\
Swing time (s) & 0.603 & 0.554 & 0.073 & & 0.473 & 0.460 & 0.485 \\
\hline
\end{tabular}

${ }^{*} \mathrm{p}<0.05$.

Table 3. Mean difference in CWT and spatiotemporal symmetry (paretic/non-paretic)

\begin{tabular}{lccc}
\hline \multicolumn{1}{c}{ Variable } & Pre-intervention & Post-intervention & p-value \\
\hline CWT (m/s) & $0.471 \pm 0.21$ & $0.558 \pm 0.23^{*}$ & $<0.001$ \\
Step-length symmetry & $1.017 \pm 0.25$ & $0.990 \pm 0.19$ & 0.720 \\
Stance-time symmetry & $0.934 \pm 0.12$ & $0.943 \pm 0.11$ & 0.770 \\
Swing-time symmetry & $1.279 \pm 0.23$ & $1.211 \pm 0.21$ & 0.109 \\
Overall temporal symmetry & $1.404 \pm 0.40$ & $1.314 \pm 0.31$ & 0.218 \\
\hline
\end{tabular}

Values are presented as mean \pm standard deviation.

CWT, Comfortable 10-m Walk Test.

${ }^{*} \mathrm{p}<0.05$. 


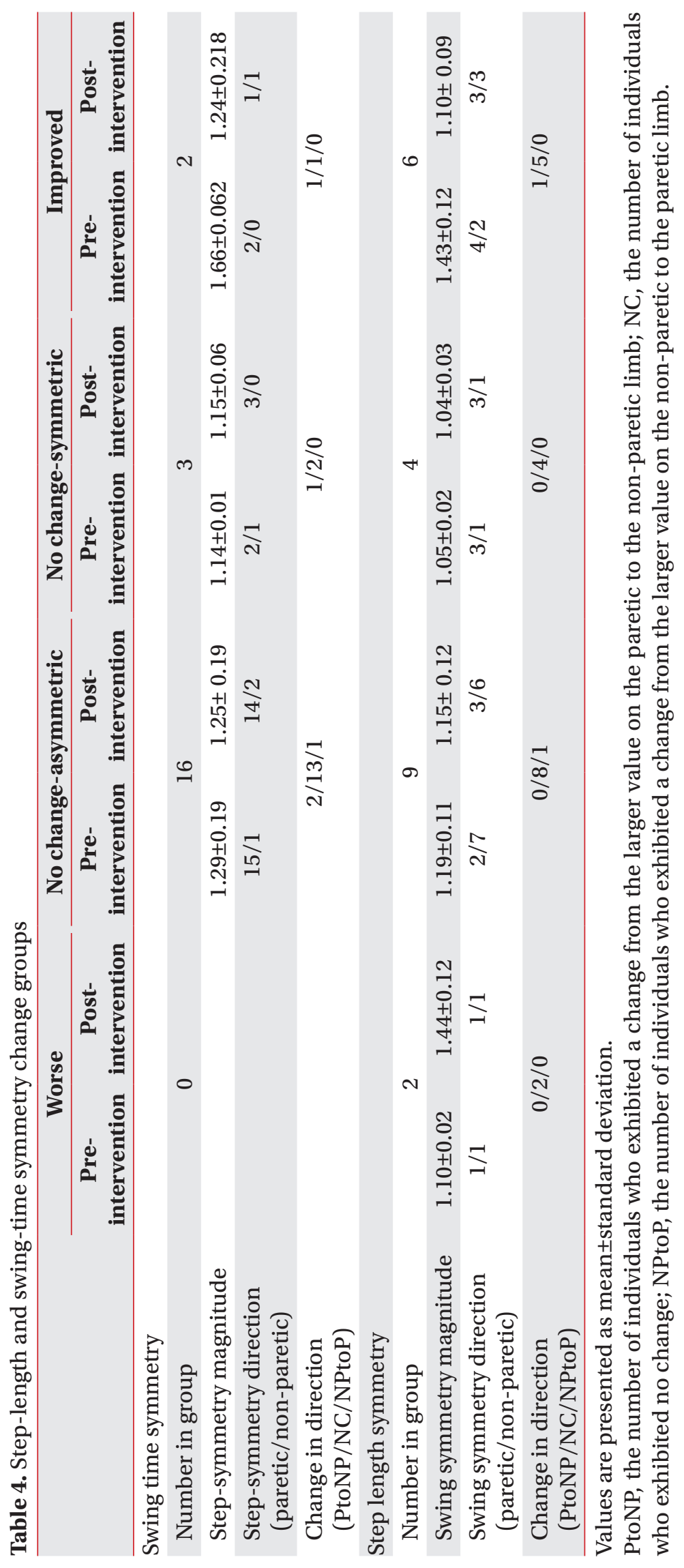


Table 5. Mean scores across time for balance and balance confidence

\begin{tabular}{lccc}
\hline & Pre-intervention & Post-intervention & p-value \\
\hline BBS & $39.66 \pm 8.63$ & $43.80 \pm 5.21^{*}$ & $<0.001$ \\
ABC & $38.39 \pm 13.46$ & $46.93 \pm 12.32^{*}$ & $<0.001$ \\
\hline
\end{tabular}

Values are presented as mean \pm standard deviation.

BBS, Berg Balance Scale; ABC, Activities-Specific Balance Confidence.

${ }^{*} \mathrm{p}<0.05$.

The mean pre-intervention CWT of $0.471 \mathrm{~m} / \mathrm{s}$ (SD= 0.21 ), changed to mean post-intervention CWT of 0.558 $\mathrm{m} / \mathrm{s}(\mathrm{SD}=0.23)$, showing statistically significant increase $(p<0.001)$ (Table 3). For step-length symmetry, stancetime symmetry, swing-time symmetry, and overall temporal symmetry, the posttest SR was closer to 1 , as compared to the pretest SR, but this was not statistically significant. The subjects were classified into 4 groups, according to the variation in swing-time symmetry ratio and step-length symmetry ratio before and after intervention (Table 4). For step length symmetry, 6 subjects were in the 'Improved' group, 13 in the 'No change' group, and 2 in the 'Worse' group. For swing time symmetry, 2 were in the 'Improved' group, 19 in the 'No change' group, and none in the 'Worse' group.

The ABC and BBS scores are shown in Table 5 as the secondary outcomes. The mean pre-intervention BBS score was $39.66(\mathrm{SD}=8.63)$, which increased to mean of $43.80(\mathrm{SD}=5.21)$ at post-intervention. The $\mathrm{ABC}$ score changed from $38.39 \pm 13.46$ to $46.93 \pm 12.32$ from pre- to post-intervention. The changes in both BBS and ABC scores were significant $(\mathrm{p}<0.001)$.

\section{DISCUSSION}

Previous studies have addressed the influence of ATT on the cardiovascular system and energy expenditure in healthy people, obese people, and patients with musculoskeletal disease, as well as in stroke patients [19-21]. Yoo et al. [8] described changes in blood pressure (BP) and heart rate (HR), before and after a single session of ATT in stroke patients. Lambert et al. [22] demonstrated blood pressure changes after ATT and land treadmill training, and Jung et al. [23] compared energy expenditure for ATT and overground treadmill use. To our knowledge, there have been only two reports on effectiveness of ATT on gait ability in stroke patients. Park et al. [24] reported improvement in balance ability before and after ATT in stroke patients. Jung et al. [23] reported that the addition of weight on the affected leg reduced unwanted flotation in chronic stroke patients during ATT, leading to increased stance stability. There have been a few studies on changes in $\mathrm{BP}, \mathrm{HR}$, energy expenditure, and balance ability in stroke patients before and after ATT. However, the present study is the first to compare gait symmetry in stroke patients by gait analysis, with assessment of changes on the BBS and ABC scales, before and after ATT.

This study evaluated the improvements in gait symmetry using spatiotemporal data from gait analysis as the primary outcome, and improvements in balance function and subjective balance confidence as the secondary outcomes. Thus, this study is the first to evaluate the efficacy of ATT in stroke patients in terms of gait spatiotemporal parameters, as well as BBS and ABC.

CWT increases in most acute and subacute stroke patients during rehabilitation, but has a weak association with spatial symmetry (step symmetry); only CWT shows a high rate of improvement after stroke [14]. In this study, CWT results increased significantly from $0.471 \mathrm{~m} / \mathrm{s}$ to $0.548 \mathrm{~m} / \mathrm{s}$. As both the stance time and swing time in the paretic leg decreased in the post-intervention period, the stride time, which is the sum of these two, also decreased, with a corresponding increase in post-intervention CWT. The stance time symmetry of the paretic and non-paretic legs changed from 0.934 to 0.943 and was closer to 1 , but the difference was not significant $(\mathrm{p}=0.770)$. Overall temporal symmetry in the paretic and non-paretic legs showed a negative correlation with motor impairment in the lower limbs [13]. This value decreased from 1.404 to 1.314 , but was not significant $(\mathrm{p}=0.218)$ in this study.

Step length spatial symmetry changed from 1.017 to 0.990 , but without statistical significance $(\mathrm{p}=0.720)$. The step length of the paretic leg increased from $0.351 \mathrm{~m}$ to $0.406 \mathrm{~m}(\mathrm{p}=0.060)$, whereas the step length of the nonparetic leg also increased significantly, from $0.353 \mathrm{~m}$ to $0.414 \mathrm{~m}(\mathrm{p}=0.024)$. Thus, the post-intervention step length symmetry was lesser than 1 . According to a report by Balasubramanian et al. [25], step length is related to temporal parameters, including swing time and stance time. Step length symmetry is an established useful measurement that can be used to evaluate propulsive force produced by the paretic leg. Longer step length is related 
to longer strides with increasing propulsive force in the pre-swing phase $[26,27]$. Step length ratio $>1$ implies increase in the step length of the paretic leg; this is assumed to be related to an increase in the propulsive force of the paretic limb.

CWT is weakly correlated with spatial symmetry. In other words, gait velocity alone can improve without corresponding recovery in spatial symmetry; such effects can be attained with adaptation to a new gait pattern, rather than with improvement in the paretic limbs [14]. We hypothesized that improved gait symmetry after ATT leads to functional improvement in the paretic limb. However, adaptation to a new gait pattern rather than functional improvement in the paretic limb, can be assumed from significant change only in CWT without significant changes in gait symmetry.

Patterson et al. [18] conducted retrospective reviewed measurements of spatiotemporal symmetry, gait velocity, motor impairment, mobility, and balance function at admission and discharge in 71 independent walking stroke inpatients undergoing conventional rehabilitation treatment. The cut-off points of swing phase symmetry and step symmetry with respect to gait were set at 1.06 and 1.08 , respectively, while the significant minimal change values for swing symmetry and step symmetry were set at 0.26 and 0.19 , respectively. When the analyzed value changed from asymmetry to symmetry, it was defined as 'improved', and improvement in swing symmetry and step symmetry was observed in 7 and 5 subjects, respectively. As the subjects in our study were categorized in the same manner, improved swing symmetry and step symmetry was observed in 2 and 6 out of the 21 patients respectively, which was a higher proportion of the total than the value of the study reported by Patterson et al. [18]. In the study by Patterson et al. [18], the mean length of hospitalization was 30.1 days, whereas the mean treatment duration was 21 days in the present study. Therefore, ATT combined with conventional therapies can be considered to have a positive effect on gait symmetry to some degree.

There were statistically significant improvements in BBS and $\mathrm{ABC}$ scores before and after the intervention. $\mathrm{A}$ BBS score showing an improvement of more than 6 points, or a posttest BBS score of more than 41 points, is interpreted as highly correlated to a reduced fall risk [15]. In this study, 8 of the 21 subjects showed an improvement of more than 6 points in the BBS, and 6 subjects whose BBS pre-intervention score was less than 41 points increased to a post-intervention score of more than 41 points, indicating that a substantial number of the subjects met the criterion.

A study by Park et al. [24], which compared the static and dynamic balance in an ATT group and a land-based training group, found improvement in the experimental group values compared with pretest values; however, the differences were not significant, compared with the landbased training group, corresponding to the results of this study.

Treadmill training encourages gait training in a more rhythmical and repetitive manner, and provides higher targets by gradually increasing the speed. In addition, Bates and Hanson [28], Hall et al. [29], and others observed that aquatic therapy offers weight support through buoyancy, and hence can improve the muscle strength and endurance, increase the ROM during gait, and improve balance ability and cardiovascular function.

Unlike land exercise against the force of gravity, aquatic exercise, with hydrostatic pressure, buoyancy, and viscosity applying forces from multiple directions, may have a positive impact on dynamic balance training. Buoyancy in water reduces the risks of falling compared with a land treadmill, enabling patients to train at a higher speed, more safely at ease $[6,21]$. Matsumoto et al. [30] also suggested that mental stability and self-confidence could improve with aquatic therapy. Therefore, positive results for CWT, BBS, and $\mathrm{ABC}$ obtained in this study were achieved by ATT, the combination of aquatic therapy and treadmill training.

The cost/benefit ratio should be addressed in the conventional rehabilitation therapy for stroke patients, including range of motion exercises, strengthening and stretching of the paretic limb to achieve independent mobility. Since such therapies all require one-on-one contact between a therapist and a patient, it is necessary to improve the temporal and economic efficiency by using mechanical equipment. Treadmill exercise, a typical mechanical activity, is used to gradually increase gait speed and reduce weight support by repeated forced use of the paretic limb during gait; thus, it is a task-oriented training-shaping exercise. BWSTT has proven to be effective in stroke patients, but requires 1-2 therapists to continuously stand by a concerned patient in order to 
maintain the torso, pelvis, and lower limbs in a proper position. Other studies on ATT showed that therapists were rarely in direct contact with patients to help in maintaining a proper posture; in most cases, verbal cues were provided, or training was continued without any verbal cues $[24,31]$. The pool was only able to accommodate one person without direct assistance or contact, and this study provided an occasional verbal cue by the physical therapist. This indicates that aquatic treadmill training might be superior to BWSTT in terms of required presence and degree of fatigue of a physical therapist.

There is no current modality known to increase gait symmetry. In a study by Patterson et al. [18], in which longitudinal changes in post-stroke spatiotemporal gait asymmetry over inpatient rehabilitation was assessed, the gait symmetry failed to improve since therapists mainly concentrated on gait velocity and gait independence, or overall gait pattern, without focusing on the correction of asymmetry; moreover, for chronic impairment, they suggested increasing difficulty to attain gait symmetry for stroke patients, as they become accustomed to a compensatory pattern. Therefore, patients and therapists should be aware of the benefits to be gained from achieving gait symmetry, and patients should make efforts to correct their gait patterns while receiving continuous feedback. ATT provides partial weight-bearing, and an environment in which overcoming water resistance forces the performance of rhythmic as well as repetitive walking on the treadmill belt. ATT could be a more effective, convenient and feasible method than other modalities, for providing continuous feedback and achieving gait symmetry. Verifying these findings would require further studies with a larger number and control subjects.

In addition, except for the treatment suspension of a patient due to an ingrown nail-related wound, there were no adverse or side effects during the present study. Possible skin injuries and fractures resulting from foot dragging were prevented by wearing ankle foot orthoses. In addition, no adverse events have been reported during ongoing aquatic training for stroke patients, whereas chest pain, dyspnea, and musculoskeletal pain were reported during ATT for other diseased groups $[21,32,33]$. We did not experience any adverse events during this study. Hence, ATT in stroke patients can be considered a safe exercise.

A limitation of this study is the small sample size. In ad- dition, this is a pre- and post-intervention comparison without a control group; if a group undergoing BWSTT or conventional therapy alone is set as a control group, the efficacy of ATT could be more clearly determined.

As mentioned above, in order to reflect multifaceted gait aspects, it is necessary to evaluate a variety of measurements, such as continuous relative phase, the moment and power of each joint at the same time, and then determine their associations with the gait symmetry.

Meanwhile, a drawback of BBS is its ceiling effect. Because of safety concerns, stroke individuals who were able to walk independently without an assistive device, were recruited for this study. Consequently, the mean pre-intervention BBS score was relatively high at 39.6 points. Specifically, there were 12 subjects with a BBS score of more than 41 points, indicating that the BBS may not have had sufficient clinical information to determine whether the balance truly improved.

Furthermore, the $\mathrm{ABC}$ was translated into Korean for use in this study. However, the ABC contains items that are somewhat unsuitable for the high percentage of subjects living in apartments in Korea (cannot be intuitively understood or imagined). For example, items such as 'Walk around the house', 'Come out of the house and walk into the parking lot', or 'Go to a shopping mall across the parking lot' are situations applicable to the United States, where the ABC scale was developed. A validated translated version more suitable for Korean environment is therefore required.

In conclusion, this study is the first to determine the efficacy of ATT on gait velocity, gait symmetry, balance, and balance confidence in stroke patients. The study found that there were improvements in the functional aspects of gait, including CWT, BBS, and ABC scores, and although not statistically significant, gait spatiotemporal symmetry also improved. The results are promising to justify further clinical studies using larger sample size and control subjects, to examine the benefits of ATT as a new modality for stroke therapy compared with other modalities.

\section{CONFLICT OF INTEREST}

No potential conflict of interest relevant to this article was reported. 


\section{REFERENCES}

1. Grysiewicz RA, Thomas K, Pandey DK. Epidemiology of ischemic and hemorrhagic stroke: incidence, prevalence, mortality, and risk factors. Neurol Clin 2008; 26:871-95.

2. O'Sullivan SB, Schmitz TJ, Fulk GD. Physical rehabilitation. 6th ed. Philadelphia: F. A. Davis Company; 2007. p. 385.

3. Wall JC, Turnbull GI. Gait asymmetries in residual hemiplegia. Arch Phys Med Rehabil 1986;67:550-3.

4. Combs SA, Dugan EL, Passmore M, Riesner C, Whipker D, Yingling E, et al. Balance, balance confidence, and health-related quality of life in persons with chronic stroke after body weight-supported treadmill training. Arch Phys Med Rehabil 2010;91:1914-9.

5. Combs SA, Dugan EL, Ozimek EN, Curtis AB. Bilateral coordination and gait symmetry after body-weight supported treadmill training for persons with chronic stroke. Clin Biomech (Bristol, Avon) 2013;28:448-53.

6. Becker BE. Aquatic therapy: scientific foundations and clinical rehabilitation applications. PM R 2009;1:85972.

7. Chu KS, Eng JJ, Dawson AS, Harris JE, Ozkaplan A, Gylfadottir S. Water-based exercise for cardiovascular fitness in people with chronic stroke: a randomized controlled trial. Arch Phys Med Rehabil 2004;85:870-4.

8. Yoo J, Lim KB, Lee HJ, Kwon YG. Cardiovascular response during submaximal underwater treadmill exercise in stroke patients. Ann Rehabil Med 2014;38: 628-36.

9. Jung T, Lee D, Charalambous C, Vrongistinos K. The influence of applying additional weight to the affected leg on gait patterns during aquatic treadmill walking in people poststroke. Arch Phys Med Rehabil 2010;91: 129-36.

10. Berg K, Wood-Dauphine S, Williams J, Gayton D. Measuring balance in the elderly: preliminary development of an instrument. Physiother Can 1989;41:30411.

11. Botner EM, Miller WC, Eng JJ. Measurement properties of the Activities-specific Balance Confidence Scale among individuals with stroke. Disabil Rehabil 2005; 27:156-63.

12. Flansbjer UB, Holmback AM, Downham D, Patten C, Lexell J. Reliability of gait performance tests in men and women with hemiparesis after stroke. J Rehabil Med 2005;37:75-82.

13. Patterson KK, Parafianowicz I, Danells CJ, Closson V, Verrier MC, Staines WR, et al. Gait asymmetry in community-ambulating stroke survivors. Arch Phys Med Rehabil 2008;89:304-10.

14. Patterson KK, Gage WH, Brooks D, Black SE, Mcllroy WE. Evaluation of gait symmetry after stroke: a comparison of current methods and recommendations for standardization. Gait Posture 2010;31:241-6.

15. Stevenson TJ. Detecting change in patients with stroke using the Berg Balance Scale. Aust J Physiother 2001; 47:29-38.

16. Blum L, Korner-Bitensky N. Usefulness of the Berg Balance Scale in stroke rehabilitation: a systematic review. Phys Ther 2008;88:559-66.

17. Lewek MD, Randall EP. Reliability of spatiotemporal asymmetry during overground walking for individuals following chronic stroke. J Neurol Phys Ther 2011; 35:116-21.

18. Patterson KK, Mansfield A, Biasin L, Brunton K, Inness EL, Mcllroy WE. Longitudinal changes in poststroke spatiotemporal gait asymmetry over inpatient rehabilitation. Neurorehabil Neural Repair 2015;29: 153-62.

19. Bressel E, Smith G, Miller A, Dolny D. Aquatic-treadmill walking: quantifying drag force and energy expenditure. J Sport Rehabil 2012:2011-0096.

20. Bressel E, Wing JE, Miller AI, Dolny DG. High-intensity interval training on an aquatic treadmill in adults with osteoarthritis: effect on pain, balance, function, and mobility. J Strength Cond Res 2014;28:2088-96.

21. Roper JA, Bressel E, Tillman MD. Acute aquatic treadmill exercise improves gait and pain in people with knee osteoarthritis. Arch Phys Med Rehabil 2013;94: 419-25.

22. Lambert BS, Greene NP, Carradine AT, Joubert DP, Fluckey JD, Riechman SE, et al. Aquatic treadmill training reduces blood pressure reactivity to physical stress. Med Sci Sports Exerc 2014;46:809-16.

23. Jung T, Ozaki Y, Lai B, Vrongistinos K. Comparison of energy expenditure between aquatic and overground treadmill walking in people post-stroke. Physiother Res Int 2014;19:55-64.

24. Park SW, Lee KJ, Shin DC, Shin SH, Lee MM, Song CH. The effect of underwater gait training on balance abil- 
ity of stroke patients. J Phys Ther Sci 2014;26:899-903.

25. Balasubramanian CK, Bowden MG, Neptune RR, Kautz SA. Relationship between step length asymmetry and walking performance in subjects with chronic hemiparesis. Arch Phys Med Rehabil 2007;88:43-9.

26. Anderson FC, Goldberg SR, Pandy MG, Delp SL. Contributions of muscle forces and toe-off kinematics to peak knee flexion during the swing phase of normal gait: an induced position analysis. J Biomech 2004;37: 731-7.

27. Goldberg SR, Anderson FC, Pandy MG, Delp SL. Muscles that influence knee flexion velocity in double support: implications for stiff-knee gait. J Biomech 2004;37:1189-96.

28. Bates A, Hanson N. Aquatic exercise therapy. Philadelphia: Saunders; 1996.

29. Hall J, Macdonald IA, Maddison PJ, O'Hare JP. Cardiorespiratory responses to underwater treadmill walking in healthy females. Eur J Appl Physiol Occup Physiol
1998;77:278-84.

30. Matsumoto I, Araki H, Tsuda K, Odajima H, Nishima S, Higaki $Y$, et al. Effects of swimming training on aerobic capacity and exercise induced bronchoconstriction in children with bronchial asthma. Thorax 1999;54:196201.

31. Stevens SL, Caputo JL, Fuller DK, Morgan DW. Effects of underwater treadmill training on leg strength, balance, and walking performance in adults with incomplete spinal cord injury. J Spinal Cord Med 2015;38:91101.

32. Mehrholz J, Kugler J, Pohl M. Water-based exercises for improving activities of daily living after stroke. Cochrane Database Syst Rev 2011;(1):CD008186.

33. Greene NP, Lambert BS, Greene ES, Carbuhn AF, Green JS, Crouse SF. Comparative efficacy of water and land treadmill training for overweight or obese adults. Med Sci Sports Exerc 2009;41:1808-15. 